\title{
Oncoplastic Surgery in Breast Cancer: Don't Forget the Boost!
}

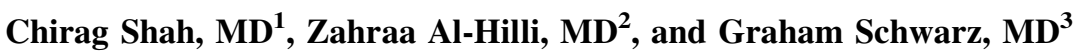 \\ ${ }^{1}$ Department of Radiation Oncology, Taussig Cancer Institute, Cleveland Clinic, Cleveland, OH; ${ }^{2}$ Department of General \\ Surgery, Digestive Disease and Surgery Institute, Cleveland Clinic, Cleveland, OH; ${ }^{3}$ Department of Plastic Surgery, \\ Dermatology and Plastic Surgery Institute, Cleveland Clinic, Cleveland, $\mathrm{OH}$
}

A growing focus on cosmetic outcomes for women undergoing breast-conserving therapy has emerged, spurring the development of oncoplastic/partial breast reconstruction surgical techniques. Oncoplastic techniques can be offered to a large number of patients and include a variety of procedures. Local breast tissue rearrangements such as glandular advancement or rotation flaps can be employed for smaller defects, whereas, mastopexy and reduction mammoplasty may be offered for larger resections and in patients with larger-volume breasts. ${ }^{1}$ The goals of these techniques are multifold and include restoration of an aesthetic breast contour and optimization of breast symmetry, while enabling the surgeon to obtain generous margins during oncologic resection. ${ }^{2-6}$ Furthermore, patients with macromastia or significant ptosis undergoing radiotherapy may benefit from both reduced toxicity and improved function through relief of symptomatic macromastia. Taken together, these benefits translate into improvements in breast-related quality of life in women with breast cancer. However, one of the challenges associated with the increased use of oncoplastic techniques is incorporating the tumor bed boost that often accompanies whole-breast irradiation (WBI) following breast-conserving surgery. As such, the questions that have emerged include (1) what is the role of tumor bed boost following breast-conserving surgery, and (2) how can oncoplastic surgery be integrated into a paradigm that includes tumor bed boosts?

(C) Society of Surgical Oncology 2018

First Received: 24 March 2018;

Published Online: 15 June 2018

C. Shah, MD

e-mail: shahc4@ccf.org

\section{WHY IS A TUMOR BED BOOST IMPORTANT IN THE MANAGEMENT OF BREAST CANCER?}

Several randomized trials have been performed evaluating the role of tumor bed boost, which remains an integral part of current breast cancer radiotherapy paradigms for women undergoing breast conservation, as noted by recent evidence-based guidelines and clinical trials. ${ }^{7-12}$ EORTC 22881 randomized over 5300 women with stage I/II breast cancers to the addition of a boost (16 Gy, lumpectomy cavity $+1.5 \mathrm{~cm}$ ) or no boost for patients with negative surgical margins. Initial outcomes at 10 years demonstrated a reduction in local recurrence $(10.2 \%$ vs. $6.2 \%, \mathrm{p}<.0001$ ). The relative benefit in rates of local recurrence were maintained across all age groups; however, the absolute local recurrence benefit was diminished with increasing age (age $<40$ years: 24 vs. 13\%; 41-50 years: 12 vs. $8 \%$; 51-60 years: 7 vs. $4 \%$; $>60$ years: 7 vs. $4 \%$ ), while the addition of a boost was associated with increased fibrosis. ${ }^{7}$ Twenty-year outcomes from this study were recently published, with boost demonstrating a persistent reduction in ipsilateral breast tumor recurrence (IBTR) [17 vs. $12 \%]$, with no plateau and increasing benefit to boost noted over time, although no breast cancer or overall survival advantage was noted. ${ }^{11}$ Factors associated with IBTR included young age, the presence of ductal carcinoma in situ (DCIS), and highgrade disease (first 5 years of follow-up). ${ }^{11}$ With respect to DCIS, Moran et al. recently published a pooled analysis demonstrating a reduction in IBTR with the addition of a tumor bed boost at 5,10 and 15 years (3.7 vs. $2.9 \%, 7.5$ vs. $5.9 \%, 12$ vs. $8.4 \%$, respectively), with an increasing benefit noted with longer follow-up. Additionally, boost was associated with a reduction in IBTR independent of age and tamoxifen use. ${ }^{12}$ Taken together, these data support the need to consider tumor bed boost in most women 
undergoing breast-conserving surgery. While there are questions regarding the magnitude of benefit in the modern era, given updated surgical and systemic therapy approaches, there are limited data to support eliminating a tumor bed boost at this time. Additionally, while one may consider larger margins in an effort to forgo a boost, there remains a paucity of data supporting this approach. Further study and updated data are needed to best identify those patients requiring a tumor bed boost.

\section{INCORPORATION OF THE TUMOR BED BOOST WITH AN ONCOPLASTIC SURGICAL PARADIGM: BEST PRACTICES}

Importantly, a preoperative, multidisciplinary discussion with the oncologic surgeon, reconstructive surgeon, and radiation oncologist is crucial in order to identify the necessity of and the target for a boost when oncoplastic techniques are anticipated. While surgical clips can be placed to delineate the surgical bed, tissue rearrangement can alter the original positioning, limiting their ability to serve as fiducial markers of the lumpectomy cavity and atrisk tissue. However, this may not be an issue with small resections and limited tissue rearrangement. Perhaps most important is a multidisciplinary debriefing following the oncoplastic procedure. This is a critical measure that allows the radiation oncologist to understand the postoperative anatomy so they may delineate the areas at highest risk for incorporation into the target boost volume.

An alternative treatment strategy is an intraoperative radiation therapy (IORT) boost following lumpectomy but prior to tissue rearrangement. Multiple studies have been published documenting the safety and efficacy of IORT boost, although limited data are available in the setting of oncoplastic surgery. ${ }^{13,14}$ This approach would allow for completion of the boost prior to any tissue rearrangement or cavity alteration, with WBI to follow. This technique can be used when oncoplastic surgery follows lumpectomy in one procedure, or with a staged approach where, following initial resection, the patient is brought back to the operating room for IORT followed by their oncoplastic procedure. Challenges with such an approach include the need for IORT equipment and sequencing. This strategy would not be ideal if a patient requires chemotherapy as there would be a significant delay between boost and WBI (although the sequence can be flipped with respect to systemic therapy and radiotherapy). ${ }^{15}$ Additionally, there are limitations with respect to IORT and cavity size (up to $5 \mathrm{~cm}$ ), which may limit feasibility beyond smaller resection cavities. Finally, with low-energy IORT, approximately $25 \%$ of prescription dose is administered at $1 \mathrm{~cm}$ from the lumpectomy cavity. This is different than other boost techniques (electrons, photons, brachytherapy, electron IORT), which usually deliver $100 \%$ of the prescription at $1 \mathrm{~cm}$ or greater; however, IORT boost series have confirmed the safety and efficacy of the technique and IORT represents a technique that allows for the easiest incorporation of boost into oncoplastic procedures. ${ }^{13,14,16}$

Placement of a device (e.g. Biozorb; Focal Therapeutics, Aliso Viejo, CA, USA) to mark the location and extent of the lumpectomy cavity is yet another strategy. ${ }^{17}$ This would also potentially allow for sequencing such that chemotherapy could precede radiotherapy, and for offering boost at centers without IORT. Challenges with this approach include the need to preserve not only the cavity but a margin of normal breast tissue around the cavity, such that the boost target volume is comparable to standard boost volumes. Additionally, this approach requires the placement of a device into the patient, which adds additional cost to the procedure. Finally, inclusion of such a device to maintain the cavity may limit the ability to close the defect using oncoplastic techniques at the time of surgery. As such, this is not considered an ideal approach to be incorporated with oncoplastic surgery when using techniques that require such approximation.

A third strategy would be to consider a delayed oncoplastic approach where the initial surgery would consist of a lumpectomy; boost could be delivered perioperatively using IORT or other partial breast approaches, including interstitial or applicator brachytherapy, with planned oncoplastic surgery to follow and then WBI. This allows for clinicians who do not have IORT to use an alternative partial breast boost technique; however, it does add an additional surgical procedure and would also face the same challenges as an IORT boost for those patients requiring systemic therapy. Nonetheless, this would likely increase the rate of wound complications and is therefore a suboptimal approach compared with the techniques mentioned earlier. In addition, this would only work with some planned oncoplastic procedures (ex. mammoplastic reduction without additional tissue rearrangement) as the delivery of radiation therapy would complicate subsequent surgery to the tumor bed. An option for such approaches would be a delayed approach for contour deformity (regional flaps, fat grafting), relying on distant donor sites.

Finally, while not a solution, a consideration can also be made to forgo oncoplastic surgery in those patients who may derive the greatest benefit to a tumor bed boost (younger age, presence of associated DCIS, high-grade disease) and are not able to receive a boost in conjunction with their proposed oncoplastic procedure. An alternative would be to omit a tumor bed boost for those patients desiring oncoplastic surgery who derive the least benefit 
(older age, no associated DCIS, low grade). ${ }^{18}$ However, the key remains appropriate patient risk stratification and communication between surgeons and radiation oncology.

As oncoplastic approaches to breast-conserving cancer treatment are increasingly adopted, individualized treatment plans with respect to surgical planning and radiation therapy are required. The most important measure ensuring optimal outcomes is perioperative multidisciplinary communication and enhanced coordination between the oncologic surgeon, reconstructive surgeon, and radiation oncologist.

DISCLOSURE Chirag Shah is a consultant for Impedimed Inc. Zahraa Al-Hilli and Graham Schwarz declare no conflicts of interest.

\section{REFERENCES}

1. Weber WP, Soysal SD, El-Tamer M, et al. First international consensus conference on standardization of oncoplastic breast conserving surgery. Breast Cancer Res Treat. 2017;165:139-49.

2. Clough KB, van la Parra RFD, Thygesen HH, et al. Long-term results after oncoplastic surgery for breast cancer: a 10-year follow-up. Ann Surg. 2018;268(1):165-71.

3. Clough KB, Ihrai T, Oden S, et al. Oncoplastic surgery for breast cancer based on tumour location and a quadrant-per-quadrant atlas. Br J Surg. 2012;99:1389-95.

4. Carter SA, Lyons GR, Kuerer HM, et al. Operative and oncologic outcomes in 9861 patients with operable breast cancer: singleinstitution analysis of breast conservation with oncoplastic reconstruction. Ann Surg Oncol. 2016;23:3190-8.

5. De La Cruz L, Blankenship SA, Chatterjee A, et al. Outcomes after oncoplastic breast-conserving surgery in breast cancer patients: a systematic literature review. Ann Surg Oncol. 2016;23:3247-58.

6. Crown A, Wechter DG, Grumley JW. Oncoplastic breast-conserving surgery reduces mastectomy and post-operative reexcision rates. Ann Surg Oncol. 2015;22:3363-8.

7. Bartelink H, Horiot JC, Poortmans PM, et al. Impact of a higher radiation dose on local control and survival in breast-conserving therapy of early breast cancer: 10-year results of the randomized boost versus no boost EORTC 22881-10882 trial. J Clin Oncol. 2007;25:3259-65.

8. Romestaing P, Lehingue Y, Carrie C, et al. Role of a 10-Gy boost in the conservative treatment of early breast cancer: results of a randomized clinical trial in Lyon, France. J Clin Oncol. 1997;15:963-8.

9. Polgar C, Fodor J, Orosz Z, et al. Electron and high-dose-rate brachytherapy boost in the conservative treatment of stage I-II breast cancer first results of the randomized Budapest boost trial. Strahlenther Onkol. 2002;178:615-23.

10. RTOG 1005 Protocol Information. https://www.rtog.org/Clinica 1Trials/ProtocolTable/StudyDetails.aspx?study $=1005$.

11. Vrieling C, van Werkhoven E, Maingon P, et al. Prognostic factors for local control in breast cancer after long-term follow-up in the EORTC boost vs. no boost trial: a randomized clinical trial. JAMA Oncol. 2017;3:42-8.

12. Moran MS, Zhao Y, Ma S, et al. Association of radiotherapy boost for ductal carcinoma in situ with local control after wholebreast radiotherapy. JAMA Oncol. 2017;3:1060-8.

13. Vaidya JS, Baum M, Tobias J, et al. Long-term results of targeted intraoperative radiotherapy (TARGIT) boost during breast-conserving surgery. Int J Radiat Oncol Biol Phys. 2011;81:1091-7.

14. Fastner G, Sedlmayer F, Mertz F, et al. IORT with electrons as boost strategy during breast conserving therapy in limited stage breast cancer: long term results of an ISIORT pooled analysis. Radiother Oncol. 2013;108:279-86.

15. Bellon JR, Come SE, Gelman RS, et al. Sequencing of chemotherapy and radiation therapy in early-stage breast cancer: updated results of a prospective randomized trial. J Clin Oncol. 2005;23:1934-40.

16. Shah $\mathrm{C}$, Wobb J, Khan A. Intraoperative radiation therapy in breast cancer: still not ready for prime time. Ann Surg Oncol. 2016;23:1796-8.

17. Ward RC, Wiggins DL, Donegan L, et al. Biozorb tissue marker as seen on multiple imaging modalities. Breast $J$. 2018;24(2):207-9.

18. Smith BD, Bellon JR, Blitzblau R, et al. Radiation therapy for the whole breast: executive summary of an American Society for Radiation Oncology (ASTRO) evidence-based guideline. Pract Radiat Oncol. 2018;8(3):145-52. 